\title{
¿Soy lo que ves? Microagresiones capacitistas y visibilidad de la discapacidad
}

\author{
Am I what you think? Ableist microaggressions and the visibility of \\ disability
}

\begin{abstract}
Resumen
Las microagresiones, como expresión de discriminación dirigida hacia personas con discapacidad, son un fenómeno social que cuenta con escasa atención en nuestro entorno. El presente trabajo supone una aproximación cuantitativa hacia la experiencia de microagresiones capacitistas, atendiendo tanto a su frecuencia como a la diversidad de sus manifestaciones. Presentamos los resultados obtenidos, bajo método de encuesta $(n=681)$, atendiendo al grado de visibilidad de la discapacidad. Nuestros datos respaldan la implicación de dicho factor como determinante de la experiencia de microagresiones capacitistas. Las personas con discapacidades visibles presentan niveles significativamente más altos en cuanto a la frecuencia de microagresiones. Para las personas con discapacidades semi visibles o invisibles, las microagresiones más habituales están relacionadas con el cuestionamiento de las necesidades de apoyo o la minimización de la discapacidad. La interpretación desde un sesgo binario, que identifica al otro como normal o anormal, sustenta multitud de prácticas producidas por el sistema capacitista.
\end{abstract}

\section{Palabras clave}

Discapacidades visibles, discapacidades ocultas, capacitismo, microagresiones, discriminación, sesgo binario.

\begin{abstract}
Microaggressions, as an expression of discrimination of people with disabilities, are a social fact little studied. This work made a quantitative approach to the experience of ableist microaggressions, attending to the frequency and diversity of these manifestations. We present survey results $(n=681)$, attending to the degree of visibility of the disability. Our data support that this factor is determinant in the experience of capacitive micro-aggressions. The visible disabilities present higher levels in terms of the frequency of microaggressions. For semi-visible or invisible disabilities, the most common microaggressions are related to the need for support or the minimization of disability. The interpretation from a binary bias, which identifies the other as normal or abnormal, supports many practices produced by the ableist system.
\end{abstract}

\section{Keywords}

Visible disabilities, hidden disabilities, ableism, microaggression, discrimination, binary bias.

\section{Eva Moral Cabrero \\ <evamoral@usal.es>}

Universidad de Salamanca. España

\section{Agustín Huete García} <ahueteg@usal.es>

Universidad de Salamanca. España

\section{Emiliano Díez Villoria <emid@usal.es>}

Universidad de Salamanca. España

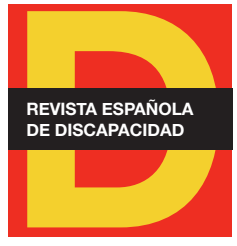

Para citar:

Moral, E. et al. (2020). “¿Soy lo que ves? Microagresiones capacitistas y visibilidad de la discapacidad". Revista Española de Discapacidad, 8(2), pp. 7-31.

Doi: <https://doi.org/10.5569/23405104.08.02.01>

Fecha de recepción: 15-11-2020 Fecha de aceptación: 23-11-2020 


\section{Introducción ${ }^{1}$}

La discapacidad como hecho social se activa y sostiene sobre un conjunto de valores y normas que se transmiten y reproducen a través de una gran variedad de elementos: discursivos, legislativos y administrativos, institucionales, etc. (Foucault, 1991: 128-129). Estos estructuran, a su vez, un sistema capacitista que diferencia dos campos en los que se ubican, por un lado, quienes cumplen con los estándares normativos históricamente definidos por el modelo médico - de la discapacidad en este caso - y quienes, alejados del promedio, son ubicados en los márgenes de la sociedad a través de prácticas que, de manera intencional o no, desarrollan individuos e instituciones desde el prejuicio y el ejercicio del poder de un grupo sobre otro considerado inferior (Campbell, 2001; Hutcheon y Wolbring, 2012).

El estándar corporal, base del capacitismo, genera esa "red de creencias, procesos y prácticas que produce un tipo particular de ser (...) perfecto, típico de la especie y, por lo tanto, esencial y completamente humano. La discapacidad entonces se presenta como un estado disminuido de ser humano" (Campbell, 2001: 44). La definición de lo que una persona válida es, tiene lugar desde el privilegio de la hegemonía de los cuerpos funcionalmente normativos, y es desde esta incorporación que se producen las prácticas que lo sustentan. Esta discriminación amplia impregna, también, procesos relacionados con el desarrollo de la propia identidad (Forber-Pratt et al., 2017; Gill, 1997), a través de la interiorización de los mensajes y agresiones recibidos de manera reiterada, ya sea explícita o implícitamente (Deal, 2007; Dirth y Branscombe, 2019; Dunn, 2019; Friedman, 2016, 2017; Harder et al., 2019).

La discapacidad, puede ser así explicada como una construcción cultural, en la que el cuerpo "normal" o "normativo" - es decir el de la mayoría de las personas consideradas "normales" - es culturalmente considerado más valioso que el cuerpo de las personas con discapacidad, lo cual se traduce en privilegios para los primeros, y desventajas para los segundos (Marín, 2013).

En este trabajo tratamos de profundizar, mediante resultados empíricos, en el estudio de la discriminación capacitista en España, a partir de una manifestación muy concreta y poco explorada, como son las microagresiones capacitistas. Específicamente nos centraremos en el peso que la visibilidad de la discapacidad tiene como posible factor determinante en la experiencia de microagresiones capacitistas, así como en el contenido ideológico desde el que se perpetúan dichas acciones.

\footnotetext{
1. Agradecimientos: el presente trabajo de investigación ha podido realizarse gracias a la participación generosa y voluntaria de todas y cada una de las personas con discapacidad que cumplimentaron la encuesta. También, a través de la inestimable colaboración, para su difusión, por parte de diversas entidades e instituciones comprometidas con el avance de los derechos de las personas con discapacidad. A quienes desinteresadamente, desde el convencimiento de que el estudio del capacitismo resulta necesario para el cambio de paradigma social a través del que se entiende e interpreta la discapacidad, colaboraron en la adaptación del instrumento. Agradecemos también el trabajo de revisión, cuyas aportaciones y sugerencias han mejorado sin duda el manuscrito.
} 


\section{Marco conceptual: microagresiones capacitistas}

El capacitismo, como sistema de desigualdad, se formula de maneras diversas, siendo las microagresiones una de sus expresiones. Como se ha señalado, estas microagresiones tienen presencia y alteran todos los ámbitos de la vida; se dan en instituciones como la familia, la escuela o las propias organizaciones de la discapacidad (Oliver, 1990) y se perpetran de maneras más o menos disimuladas (Bell, 2015; Bogart y Dunn, 2019; Campbell, 2008, 2009; Wolbring, 2008). Así, como mecanismos de discriminación, las microagresiones interrumpen o imposibilitan el disfrute de derechos, así como el desarrollo de una vida en igualdad de condiciones que el resto de la población y suponen un impacto negativo en el desarrollo de la identidad y el autoconcepto de las personas que las reciben. Por ello, resulta fundamental conocer cómo se formulan, cuál es su origen y reflexionar sobre estrategias para contrarrestarlas y prevenir su reproducción (Dirth y Branscombe, 2018, 2019; Dunn y Andrews, 2015; Forber-Pratt et al., 2017; Harder et al., 2019; Keller y Galgay, 2010).

Como ocurre con el capacitismo, y apuntan autoras como Bell (2015) o Schaff (2017), la literatura sobre microagresiones se nutre de los trabajos sobre población racializada (Grier-Reed, 2010; Mwangi et al., 2018; Solorzano et al., 2000; Yosso et al., 2009); también en el estudio de las microagresiones sexistas (Chambers, 2011; Sue, 2010). Los trabajos que se centran en las dirigidas a personas con discapacidad son aún escasos (Conover et al., 2017) y, si bien tienen puntos comunes con otras minorías, presentan otros específicos, como los identificados en el estudio de Keller y Gallgay (2010).

Las microagresiones se definen como "conductas verbales o de comportamiento breves que comunican desprecio o insultan a alguien por ser miembro de un grupo social" (Pierce et al., 1978). Se consuman en las interacciones diarias por personas cercanas o desconocidas y pueden tener un carácter ambiguo, lo que dificulta su identificación, respuesta y denuncia. Cabe señalar que, a pesar de utilizar el prefijo micro-, de acuerdo con Wells (2013), éste no hace referencia a la dimensión del daño que puedan suponer para la víctima - todo lo contrario, dada su repercusión y alta frecuencia-, pero permite la diferenciación en relación a delitos de odio o agresiones que, entendidas como macro, puedan suponer un daño físico. Como expone la autora, quienes incurren en microagresiones capacitistas suelen hacerlo a través del lenguaje y, generalmente, sin intención de dañar a quienes las reciben.

\subsection{El estudio de las microagresiones capacitistas}

El trabajo realizado por Keller y Galgay (2010) exploró, a través de dos grupos focales formados por un total de 12 participantes con discapacidad, las particularidades en las microagresiones que reciben con mayor frecuencia. Tras el análisis de la información recogida de ambos grupos, así como en la grabación de una sesión informativa posterior, proponen una agrupación de las microagresiones capacitistas en los siguientes dominios:

- Negación de identidad: ya sea (1) personal, que supone la invisibilidad o rechazo de cualquier experiencia o identidad de una persona distinta a la discapacidad, o (2) de negación de la experiencia de la discapacidad: (i) minimizando situaciones de discriminación, (ii) dando a entender que se conoce la solución y (iii) negando la discapacidad misma. 
- Negación de privacidad: tiene lugar cuando se demanda información sobre la situación de discapacidad de manera explícita o sutil, obviando las reglas sociales y la propia ubicación en un rango de confianza necesario que permita una comunicación libre.

- Indefensión: se basa en el entendimiento de que cualquier persona con discapacidad necesita ayuda en prácticamente cualquier situación.

- Ganancia secundaria: supone una agenda oculta en la interacción con una persona con discapacidad, bien para conseguir un reconocimiento social de su acción o para sentirse mejor consigo mismo.

- Efecto de extensión o propagación: la presencia de una discapacidad conlleva una atribución relacionada con otras áreas funcionales, ya sea como virtud o desventaja.

- Condescendencia o infantilización: alude al trato como eternos menores a personas de distintas edades y diversas necesidades de apoyo vinculadas a cualquier discapacidad, haciendo, explicando o decidiendo por ellas ante cualquier situación; también mostrando admiración ante su autonomía.

- Ciudadanía de segunda clase: tiene lugar cuando se cuestionan los ajustes o apoyos orientados a la consecución de la igualdad de oportunidades como irracionales, injustificados o molestos. Puede expresarse como (1) evitación, ignorando la presencia de la persona con discapacidad para no interactuar con ella; (2) carga, cuando las adaptaciones o las medidas de discriminación positiva se estiman costosas o requieren de demasiado tiempo, esfuerzo o recursos, y (3) del entorno, diferenciando espacios o directamente negando su presencia.

- Desexualización: se entiende a las personas con discapacidad como seres asexuales, negándoles la posibilidad de ser sujetos de deseo o de desarrollar actividad sexual alguna.

Además de estos ocho dominios, Keller y Galgay proponen dos menos desarrolladas: la erotización², que supone la hipersexualidad a una persona basándose únicamente en su discapacidad; y la intervención espiritual, que recoge la experiencia de personas que rezan motivadas por la presencia de otras con discapacidad, cosifica a los sujetos hacia quienes se dirigen.

\subsection{Hacia una propuesta operativa}

Basándose en la propuesta de Keller y Galgay, Kristin J. Conover (2015) realizó un trabajo enfocado al desarrollo y validación de una Escala de Microagresiones Capacitistas (AMS por sus siglas en inglés) dirigida a personas con impedimento físico según la Americans With Disabilities Act ${ }^{3}$ (ADA en adelante), así como la identificación de dimensiones de agrupación a través de la realización de los análisis factoriales exploratorios pertinentes. Si bien el estudio cuenta con una revisión posterior (Conover et al., 2017), el proceso de elaboración de la escala final, formada por 20 elementos, se explicita con mayor detalle en esta primera propuesta.

\footnotetext{
2. Se emplea el término "erotización" como corrección a una posible errata en el utilizado en el texto original "exoticization" que cabría traducir como "exotización", por adecuarse más al contenido que esta dimensión representa.

3. Recordamos que la ADA (United States, 2009) contempla en su Título 42, Sección 12102, una concepción de la discapacidad física más amplia que la que manejamos en nuestro contexto.
} 
La investigadora planteó tres estudios diferenciados: un primer estudio, cuyo objetivo fue el desarrollo propio de la escala y un análisis factorial exploratorio; un segundo estudio centrado en el análisis factorial confirmatorio, también sobre la confiabilidad y la validez de la escala, y un tercero, en el que no nos detendremos, sobre el impacto de las microagresiones capacitistas en relación con el apoyo social percibido por minorías sexuales con discapacidad.

La AMS se lanzó a través de una plataforma online y recogió una muestra final formada por 1392 personas. Como resultado de los distintos análisis factoriales exploratorios realizados, así como los análisis factoriales confirmatorios, la autora propuso un modelo de cuatro factores que recogen de la siguiente manera las distintas dimensiones propuestas por Keller y Galgay:

- Desamparo, como el primer factor y con una representación de 5 ítems, que corresponden a los dominios de indefensión, condescendencia, ganancia secundaria y negación de identidad personal como una de las variaciones del dominio de negación de identidad de Keller y Galgay.

- Minimización, como segundo factor y 3 ítems, recoge la variación de negación de la experiencia, perteneciente al dominio de negación de identidad de Keller y Galgay.

- Negación de privacidad, como tercer factor y 5 ítems. Engloba las cualidades de infantilización, efecto de extensión y negación de identidad personal de la propuesta de Keller y Galgay.

- Otrerización, como cuarto factor con 7 ítems, que corresponde a los elementos de desexualización, ciudadanía de segunda clase y efecto de extensión de Keller y Galgay.

En relación con la explotación de los datos, de cara a determinar posibles diferencias significativas de respuesta considerando diferentes factores, los resultados obtenidos en el trabajo se relacionan con los de otros estudios. En cuanto al tipo de discapacidad, como se ha comentado, Conover asumió la clasificación de impedimento físico recogida en la ADA, desestimando aquellas que se engloban dentro de lo que recoge en la categoría de impedimento mental. Realizó también análisis de varianza (ANOVA entre sujetos) con el fin de explorar las diferencias significativas que según el tipo de discapacidad pudieran existir.

Los resultados para la muestra de personas con discapacidad visual $(n=136)$, lesión cerebral $(n=19)$, discapacidad auditiva $(n=106)$, discapacidad física $(n=179)$, enfermedad crónica $(n=228)$ y quienes informan su discapacidad dentro de la categoría "otra" $(n=10)$ no demostraron diferencias significativas. Por el contrario, la visibilidad de la discapacidad apareció como indicador en cuanto a la frecuencia de la experiencia de microagresiones, siendo el grupo de participantes con una discapacidad visible $(n=135)$ el que mostró niveles significativamente más altos ( $M=41.74)$ que cualquiera de los otros dos, formados por quienes refirieron una discapacidad semi visible $(n=310$ y $M=35.05)$ y quienes la identifican como invisible $(n=354$ y $M=29.85)$.

La frecuencia de las microagresiones varía según algunas peculiaridades de la discapacidad, pero también la forma en la que se expresan. Así lo demuestran estudios como el realizado por Nario-Redmond et al. (2019) sobre distintos tipos de capacitismo, que las autoras identifican como: (1) benévolo o de inspiración, (2) hostil o (3) ambivalente, que incluye comportamientos paternalistas o condescendientes, celosos o envidiosos, deshumanizantes u objetivantes y basados en el miedo a la discapacidad. De todos ellos, el paternalismo aparece como el más arraigado, pues es el tipo de microagresión experimentada con mayor asiduidad por la muestra del estudio. Sus resultados reflejan, además, diferencias significativas según el 
grado de visibilidad de la discapacidad en el mismo sentido que las encontradas en el estudio de Conover. Aquellas relacionadas con un trato infantilizado son más frecuentes en población con discapacidades visibles, mientras que la invalidación o el cuestionamiento de las necesidades de apoyo se dirigen con mayor frecuencia hacia quienes tienen una discapacidad oculta o menos evidente.

En otro trabajo, Kattari et al. (2018) analizaron las consecuencias que el capacitismo supone para personas con discapacidades no visibles. Apareció, de manera recurrente, la figura de "la policía del cuerpo", ejercida por familiares, amistades y personas cercanas, así como por desconocedores de la situación de discapacidad personal, que fiscalizan la solicitud o la necesidad del uso de recursos y ajustes específicos, espacios reservados o dispositivos de apoyo. De esta manera, se sospecha de las personas con discapacidades que no son fácilmente identificables o resultan invisibles, y se les interpreta como "insuficientemente discapacitadas" o como personas que no se esfuerzan lo suficiente. En los últimos meses, producto de la situación de pandemia provocada por la COVID-19 y las medidas de confinamiento y restricciones a la movilidad, hemos asistido a prácticas que han evidenciado lo referido por los participantes del estudio de Kattari et al. El cuestionamiento, la acusación y la obligatoriedad de justificar - ante la denominada "policía de balcón" -, la necesidad de consideraciones específicas para población con derecho ${ }^{4}$ reconocido a flexibilizar sus limitaciones de aislamiento, movilidad y medidas de distancia social, llegaron incluso a abrir el debate de la necesidad de ser identificados con brazaletes que evitaran el insulto y la desaprobación de su comunidad (Vivas Tesón, 2020).

Estos hallazgos se confirman también por los resultados obtenidos por Rhoda Olkin et al. (2019), que exploraron la experiencia de microagresiones perpetradas hacia mujeres con discapacidades visibles e invisibles. Dentro de los estudios sociales de la discapacidad es cada vez más frecuente la literatura que considera la perspectiva de género, al entenderlo como factor fundamental en la comprensión del funcionamiento de diversos y simultáneos ejes de opresión que operan (Ashraf et al., 2018; Candela y Mulet, 2018; Harris y Wideman, 1988; Hill Collins y Bilge, 2019; Hooks, 1990; Olkin et al., 2019; Palombi, 2012; Platero y Guzmán, 2014; Purdie-Vaughns y Eibach, 2008; Schaff, 2017; Singleton, 2013; Stein y Plummer, 1994; Sue, 2010). Las mujeres con discapacidad sufren situaciones específicas relacionadas con los roles sociales asignados al género, ya sea por un efecto hiperbólico de éstos o por representar una ruptura que amplifica las situaciones de discriminación. Pero existen aportaciones desde la perspectiva de identidad estigmatizada primaria, que apuntan a que el género es un factor menos determinante en cuanto a las diferencias entre las experiencias de discriminación en hombre y mujeres con discapacidad, como parece ocurrir también en la población racializada (Levin et al., 2002; Nario-Redmond, 2010; Nario-Redmond et al., 2019; Wang et al., 2019).

En una línea similar se pueden considerar trabajos como el de Gonzales et al. (2015), dirigidos al estudio de las especificidades del capacitismo con relación a población con sufrimiento psíquico ${ }^{5}$. Sus resultados señalan microagresiones comunes a otras discapacidades como aquellas relacionadas con la invalidación de la propia experiencia, su consideración como sujetos inferiores o como parte de una ciudadanía de segunda clase. Pero también, expresiones capacitistas peculiares, como ser temidas por suponerlas personas peligrosas e inestables y para quienes la vergüenza de su situación les impone sufrimiento y silencio.

\footnotetext{
4. Instrucción de 19 de marzo de 2020 del Ministerio de Sanidad, por la que se establecen criterios interpretativos para la gestión de la situación de crisis sanitaria ocasionada por el COVID-19 (BOE núm. 76, de 20 de marzo de 2020).

5. Empleamos sufrimiento psíquico en lugar del recomendado actualmente ("problemas de salud mental") puesto que se pretende dar visibilidad al peso del lenguaje hegemónico con el que se nombra la diversidad que la discapacidad supone, en este caso, además, claramente relacionado con su origen médico que sustenta gran parte del sistema capacitista. También como respeto a la demanda en el cambio del término que surge desde el propio colectivo psiquiatrizado, anticapacitista y anticuerdista, pues si obviamente no representa a toda la población dentro de la denominación aceptada, compartimos la necesidad de desafiar el lenguaje y reflexionar sobre cómo a través de él se reproducen prácticas que, como las microagresiones mismas, formulan y expresan el capacitismo.
} 
Como propuso Goffman (1970) el componente visual de un estigma media la relación entre quien lo presenta y quienes lo perciben. Tan es así, que la inmediatez en la perceptibilidad de la diferencia opera independiente del conocimiento previo del atributo y de sus consecuencias; también del entendimiento de las implicaciones vinculadas al tipo de actividad con el que se relacionan, en el sentido de lo que se interpreta que el sujeto que presenta la distancia con lo normativo puede o no puede hacer, aquello en lo que puede o no participar, etc.

Desde la psicología social y cognitiva también se han estudiado los efectos del pensamiento categórico. En el estudio de Fisher y Keil (2018) sobre el sesgo binario, se confirma que éste no solo afecta a la manera en la que se entienden los datos, también a la toma de decisiones. Atendiendo a la producción de juicios sumarios - que realizamos como representaciones resumidas de categorías distintivas para nuevos datos o información contradictoria-, el sesgo binario, como pensamiento categórico, distorsiona la integración de la información y la formación de creencias. Interpreta la nueva información en una sola dimensión, dentro de un continuo.

Se deduce, por tanto, que existen ciertos factores que suponen una mayor exposición e implicación con este tipo de violencia, por lo que es necesario analizarlos para conocer su funcionamiento, las consecuencias de su reproducción, identificar las bases en las que se sustentan y diseñar estrategias para su afrontamiento y resistencia. En este artículo centraremos la atención en las posibles diferencias relativas al grado de visibilidad de la discapacidad como factor identificado en trabajos anteriores como crítico en lo que a la experiencia de microagresiones capacitistas se refiere.

Desde este planteamiento, la herramienta propuesta por Conover aparece como válida y consistente para la aproximación primera sobre el estado de las microagresiones capacitistas en nuestro contexto actual, cuyo estudio es incipiente (Hernández, 2018; Moscoso y Arnau, 2016; Otaola y Huete , 2019; Sanmiquel-Molinero y Pujol-Tarrés, 2020; Toboso, 2017, 2018; Toboso y Guzmán, 2010). De la misma manera, y al igual que en otros países en los que su desarrollo está más avanzado, el acercamiento desde metodologías cuantitativas es prácticamente nulo (Kattari et al., 2018; Lett et al., 2018).

\section{Método}

Los resultados que se exponen en este artículo forman parte de una investigación más amplia que se enmarca en un trabajo de tesis doctoral en proceso, que se basa, por un lado, en la propuesta de los dominios que de microagresiones capacitistas hacen Keller y Galgay (2010) y en la herramienta creada por Conover (2015), con el objetivo de ampliar el conocimiento sobre el estado de las microagresiones capacitistas en España. La primera fase del estudio se dirige al análisis de situaciones cotidianas de discriminación por discapacidad recogidas en los tuits denuncia publicados, entre septiembre y diciembre de 2017, en la red social Twitter bajo la campaña \#MeCripple ${ }^{6}$. La segunda fase del trabajo de investigación configura el estudio que se presenta en este artículo y pretende una aproximación a las microagresiones capacitistas desde una metodología cuantitativa basada en encuesta, con la pretensión de conocer la frecuencia de su

6. Moral et al. (en preparación). “\#MeCripple: Ableism, Microaggressions, and Counterspaces on Twitter in Spain”. 
experiencia, así como contrastar la posibilidad de que determinados factores como la visibilidad de la discapacidad, el inicio de esta, los apoyos requeridos o el género puedan tener en relación con la experiencia de microagresiones capacitistas.

\subsection{Técnicas e instrumentos empleados}

Para alcanzar los objetivos planteados en relación con la discriminación por discapacidad que muchas personas enfrentan en la vida cotidiana, se entendió necesario crear un cuestionario basado en la escala de Conover (2015) y ampliar la participación a personas con otras discapacidades inicialmente no contempladas en dicho trabajo, ampliando así el ámbito de interés del estudio a las microagresiones capacitistas que pueden darse en otras situaciones de discapacidad.

Dada la ampliación del objeto de estudio, y su aplicación a población residente en España, el instrumento requirió de traducción y adaptación al idioma y contexto español, y una revisión de las cuestiones relacionadas con la accesibilidad. Siguiendo los principios del diseño universal, se descartó generar dos versiones diferenciadas de la encuesta, aplicando así criterios de lectura fácil al cuestionario para todas las personas. Para ello, se contó con un panel de expertos con conocimientos sobre lectura fácil.

La encuesta se estructura en dos partes, una inicial relativa a los datos sociodemográficos y el cuestionario en el que se presentan los 20 ítems sobre microagresiones capacitistas. Se solicita a los participantes que estimen la frecuencia de la experiencia para cada una de las situaciones presentadas, en una escala tipo Likert con seis opciones de respuesta (donde 0 es nunca y 5 muchas veces). La relación de los factores propuestos en el trabajo de Conover, que puede consultarse en la tabla 3, queda representada de la siguiente manera: Factor 1: Desamparo, con 5 ítems (2, 4, 6, 12 y 13); Factor 2: Minimización, con 3 ítems (1, 19 y 20); Factor 3 Negación: con 5 ítems (3, 8, 7, 9 y 11) y el Factor 4 Otrerización, con 7 ítems (5, 10, 14, 15, 16, 17 y 18). Las puntuaciones de cada factor se calcularon con la suma de puntuaciones de los ítems pertenecientes al factor (rango variable según el factor). La puntuación total se calculó mediante la suma total de las puntuaciones de los factores (rango de 0 a 100 con valores más altos señalando mayor frecuencia de microagresiones.

\subsection{Prueba piloto}

Se realizó un pretest centrado en cuestiones de accesibilidad cognitiva, así como en la identificación de preguntas-problema, siguiendo el proceso mixto propuesto por Martín y González-Rábago (2019: 146). Revisados los datos demográficos solicitados en el cuestionario y los 20 ítems de la escala, el pretest sirvió para eliminar algunos de los primeros, relativos a la etnia, por ejemplo, o incluir preguntas sobre el grado de discapacidad reconocido o la participación en actividades desarrolladas por entidades de la discapacidad. Todos los ítems sobre microagresiones capacitistas se conservaron, resultando una primera versión de nuestro cuestionario.

Tras el proceso expuesto, se revisó la encuesta con la herramienta E2R-Helper ${ }^{7}$, se testó su adecuación para usuarios de lectores de pantalla, y se llevó a cabo un estudio piloto a través de un formulario de

7. La herramienta E2R-Helper (Asistente Lectura Fácil) es un prototipo de aplicación web (https://eapoyo-inico.usal.es/asistente-lectura-facil/) que permite obtener índices de lecturabilidad de textos en español así como una verificación detallada, frase por frase, de 25 puntos de verificación de los incluidos en la norma UNE 153101 EX de mayo de 2018, Lectura Fácil: Pautas y recomendaciones para la elaboración de documentos. Díez, E. et al. (en preparación). "E2R-Helper: Una herramienta de ayuda para la adaptación a lectura fácil”. 
Google, gracias a la colaboración de 13 personas voluntarias con edades comprendidas entre los 27 y los 55 años, de las cuales 7 eran hombres y 6 mujeres. Tras la revisión de sus comentarios, se mantuvo la estructura y preguntas de la encuesta (consultar tabla 3), haciendo una única modificación en una de ellas e incorporando algunas cuestiones a la consulta sociodemográfica, como la posibilidad de reflejar el cobro de una pensión con tener un trabajo remunerado o la distinción entre apoyos técnicos y humanos.

\subsection{Procedimiento}

La encuesta se dirige a personas con discapacidad ${ }^{8}$, mayores de 16 años y residentes en España, al menos durante los últimos cinco años. Se distribuyó el 6 de noviembre de 2019, a través del envío de correo electrónico a un censo previamente elaborado de 1046 entidades centradas en la atención de personas adultas con discapacidad y grupos de participación y acción social en redes, 61 unidades de atención a la diversidad de universidades públicas y privadas en España, así como a los 54 programas específicos de formación a personas con discapacidad intelectual que algunas de ellas ofertan. También se publicó en redes sociales y se elaboró un vídeo en lengua de signos española, con la intención de asegurar la accesibilidad de la información sobre la encuesta a la población Sorda ${ }^{9}$ signante.

El acceso a la encuesta se realizó a través de enlace URL o código QR. Iniciaba con una descripción del objetivo del estudio, así como de los requerimientos para la participación y el consentimiento informado. Se evitó el uso de términos como capacitismo o microagresiones capacitistas, presentado como un estudio dirigido a examinar situaciones cotidianas de discriminación por discapacidad. Se garantizó el anonimato de los participantes, pues no se les solicitaba ningún dato que permitiera su identificación, excepto si así lo deseaban, pudiendo proporcionar su correo electrónico de cara a ser informados de los resultados obtenidos.

Por tanto, la muestra se ha alcanzado a través de un muestreo virtual online, no probabilístico, de "bola de nieve", frecuente en investigaciones que atienden a poblaciones minoritarias o con una importante dispersión geográfica (Baltar y Gorjup, 2012). Un total de 1161 personas accedieron al cuestionario, resultando una muestra final de 681 entradas válidas tras un exhaustivo proceso de depuración, además del debido tratamiento de la matriz para la preparación de las variables en cuanto a la categorización de algunos de los elementos sociodemográficos de cara a su análisis.

\subsection{Perfil de la muestra}

El $61 \%$ de la muestra está formada por mujeres $(n=416), 37,9 \%$ hombres $(n=258)$, y un $1 \%$ que elige la opción "otro" para definirse como "género no binario" o "fluido" $(n=7)$. Presenta edades comprendidas entre los 16 y los 74 años, con una media de 41 .

Según el nivel de formación, el $4 \%$ no tiene estudios, el 18,9\% ha realizado estudios primarios o de EGB, el $17,3 \%$ ha finalizado BUP, secundaria o FP I y un $14 \%$ estudios de Bachillerato; el $45,8 \%$ tiene formación

8. Para nuestro estudio se amplía la participación de la población objetivo, no solo por tipo de discapacidad, también en relación con quienes no cuentan con un reconocimiento o valoración oficial por encima del $33 \%$, al entender que hay personas con discapacidad que no disponen de dicho certificado oficial (Jiménez y Huete, 2010).

9. Se incluye en mayúscula pues es la forma en la que dicha comunidad se diferencia de la no signante. 
universitaria o estudios de FP II. El 29,1\% de la muestra percibe una pensión, el 35, $5 \%$ se encuentra en situación de desempleo y de entre quienes están en activo (35,3\%), el 23,3\% trabaja en empleo ordinario, el $7,6 \%$ a través de la modalidad de empleo protegido y el 3,1\% trabaja por su cuenta.

Atendiendo al tipo de discapacidad, la muestra está formada por un 14,1\% de población con discapacidad auditiva; 9,3\% personas con daño cerebral; 22,2\% de enfermedad crónica; 49,3\% discapacidad física; $12,5 \%$ discapacidad intelectual; 12,8\% sufrimiento psíquico; 5,9\% espectro autista; 11,6 \% discapacidad visual, y el 5,4\% restante señala la opción "otra discapacidad".

En relación con la visibilidad de la discapacidad, el 39,4\% de la muestra declara tener una discapacidad visible o fácilmente identificable por terceras personas; en el 34,9\% de los casos la visibilidad de la discapacidad depende de la situación o de la demanda del entorno, y en un 25,7\% la discapacidad es oculta o invisible, por lo que solo si es declarada, personas ajenas al entorno próximo podrán ser conocedoras de la situación. En la tabla 1 se detalla la distribución de la variable principal de análisis según otras características sociodemográficas de la muestra.

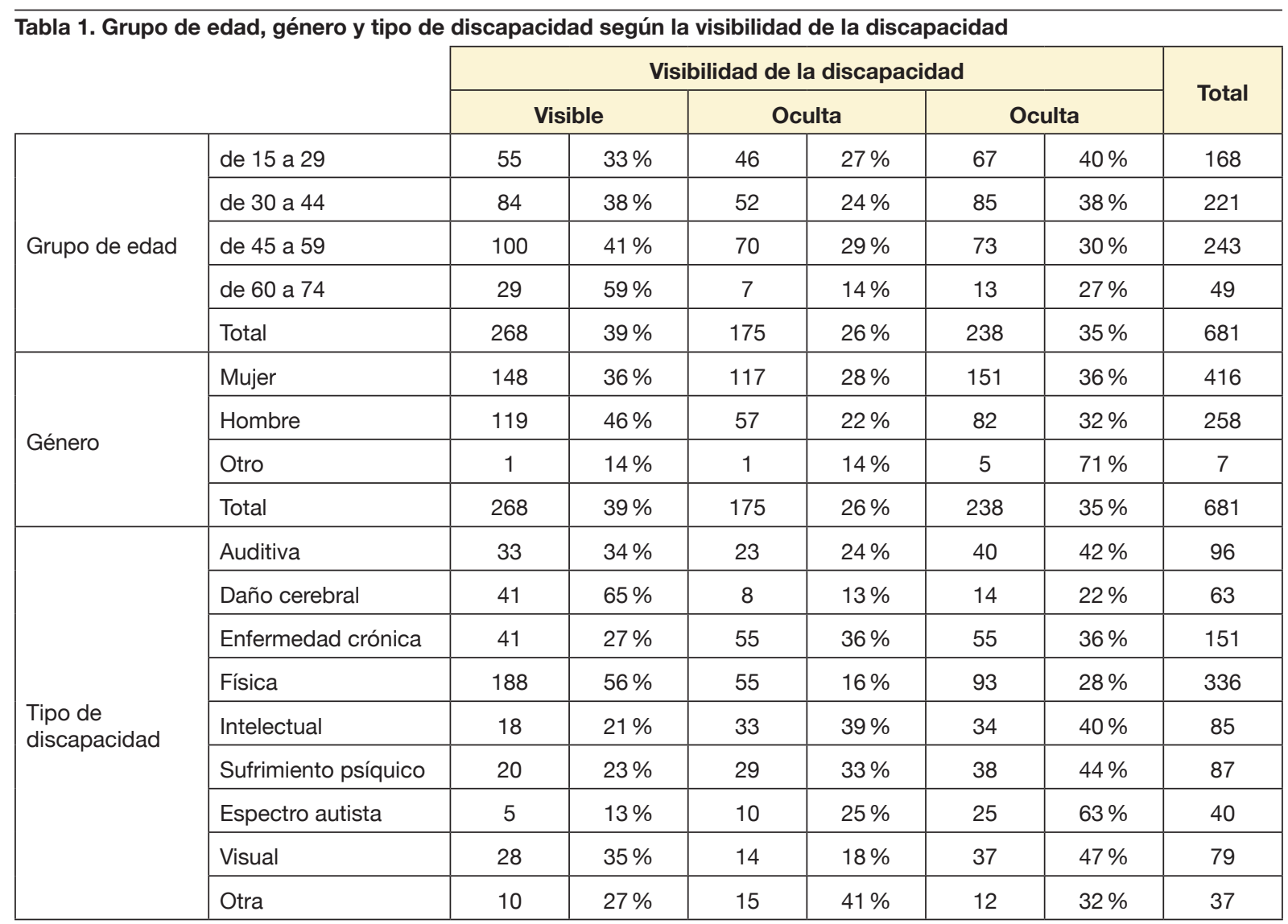

Fuente: elaboración propia. 
Otros datos específicos sobre la edad de inicio de la discapacidad, el grado de afectación o el uso de apoyos, pueden consultarse en la tabla 2.

\begin{tabular}{|c|c|c|c|c|}
\hline \multicolumn{3}{|l|}{ Variable } & Frecuencia & Porcentaje \\
\hline \multirow{11}{*}{ Discapacidad } & \multicolumn{2}{|l|}{ Auditiva } & 96 & $14,1 \%$ \\
\hline & \multicolumn{2}{|l|}{ Daño cerebral } & 63 & $9,3 \%$ \\
\hline & \multicolumn{2}{|l|}{ Enfermedad crónica } & 151 & $22,2 \%$ \\
\hline & \multicolumn{2}{|l|}{ Física } & 336 & $49,3 \%$ \\
\hline & \multicolumn{2}{|l|}{ Intelectual } & 85 & $12,5 \%$ \\
\hline & \multicolumn{2}{|l|}{ Sufrimiento psíquico } & 87 & $12,8 \%$ \\
\hline & \multicolumn{2}{|l|}{ Espectro autista } & 40 & $5,9 \%$ \\
\hline & \multicolumn{2}{|l|}{ Visual } & 79 & $11,6 \%$ \\
\hline & \multicolumn{2}{|l|}{ Otra } & 37 & $5,4 \%$ \\
\hline & \multirow{2}{*}{ Pluridiscapacidad } & No & 480 & $70,5 \%$ \\
\hline & & Sí & 201 & $29,5 \%$ \\
\hline \multirow{5}{*}{$\begin{array}{l}\text { Edad de inicio } \\
\text { discapacidad }\end{array}$} & \multicolumn{2}{|l|}{ De nacimiento } & 245 & $36 \%$ \\
\hline & \multicolumn{2}{|l|}{ Después de nacer } & 436 & $64 \%$ \\
\hline & \multicolumn{2}{|l|}{ Media } & 23,35 & - \\
\hline & \multicolumn{2}{|l|}{ Desviación } & 16,35 & - \\
\hline & \multicolumn{2}{|l|}{ Rango } & 90 & - \\
\hline \multirow{3}{*}{$\begin{array}{l}\text { Visibilidad de } \\
\text { discapacidad }\end{array}$} & \multicolumn{2}{|l|}{ La discapacidad se nota } & 268 & $39,4 \%$ \\
\hline & \multicolumn{2}{|c|}{ La discapacidad no se nota } & 175 & $25,7 \%$ \\
\hline & \multicolumn{2}{|c|}{ Se nota a veces o en algunas situaciones } & 238 & $34,9 \%$ \\
\hline \multirow{4}{*}{$\begin{array}{l}\text { Dependencia } \\
\text { reconocida }\end{array}$} & \multicolumn{2}{|l|}{ No } & 405 & $59,5 \%$ \\
\hline & \multicolumn{2}{|l|}{ Sí, Grado I } & 102 & $15,0 \%$ \\
\hline & \multicolumn{2}{|l|}{ Sí, Grado II } & 84 & $12,3 \%$ \\
\hline & \multicolumn{2}{|l|}{ Sí, Grado III } & 90 & $13,2 \%$ \\
\hline \multirow{6}{*}{$\begin{array}{l}\text { Grado de } \\
\text { discapacidad }\end{array}$} & \multicolumn{2}{|l|}{ de 0 a 32} & 79 & $11,6 \%$ \\
\hline & \multicolumn{2}{|l|}{ de 33 a 64} & 283 & $41,6 \%$ \\
\hline & \multicolumn{2}{|l|}{ de 65 o más } & 319 & $46,8 \%$ \\
\hline & \multirow{2}{*}{ Porcentaje reconocido } & Media & 53 & - \\
\hline & & Desviación & 26 & - \\
\hline & & Rango & 100 & - \\
\hline
\end{tabular}




\begin{tabular}{|c|c|c|c|}
\hline \multicolumn{2}{|l|}{ Variable } & \multirow{2}{*}{$\begin{array}{c}\text { Frecuencia } \\
357\end{array}$} & \multirow{2}{*}{$\begin{array}{c}\text { Porcentaje } \\
52,4 \%\end{array}$} \\
\hline \multirow{6}{*}{$\begin{array}{l}\text { Utiliza apoyos } \\
\text { TÉCNICOS }\end{array}$} & No & & \\
\hline & Sí & 324 & $47,6 \%$ \\
\hline & Para moverse y desplazarse & 230 & $33,8 \%$ \\
\hline & Para comunicarse & 90 & $13,2 \%$ \\
\hline & Para asearse, ir al baño, vestirse, comer, dormir, etc. & 122 & $17,9 \%$ \\
\hline & Para manejar dinero, hacer la comida, ir de compras, etc. & 113 & $16,6 \%$ \\
\hline \multirow{6}{*}{$\begin{array}{l}\text { Utiliza apoyos } \\
\text { HUMANOS }\end{array}$} & No & 484 & $71,1 \%$ \\
\hline & Sí & 197 & $28,9 \%$ \\
\hline & Asistente personal & 78 & $39,6 \%$ \\
\hline & Apoyo familiar & 30 & $15,2 \%$ \\
\hline & Intérprete de lengua de signos & 18 & $9,1 \%$ \\
\hline & Cuidadores o profesionales de centro & 16 & $8,1 \%$ \\
\hline
\end{tabular}

Fuente: elaboración propia.

\section{Resultados}

Los análisis descriptivos que exploran la relación entre las características sociodemográficas, los ítems del cuestionario y los factores propuestos por Conover aportan información relevante para la identificación de posibles indicadores de discriminación capacitista.

Como muestra la tabla 3, los ítems con puntuaciones medias más altas son el ítem $1(M=2,92)$, el $4(M=$ $2,66)$ y el $20(M=2,56)$, pertenecientes al Factor 2: Minimización y Factor 1: Desamparo. Aquellos experimentados con menor frecuencia son el ítem $10(M=1,67)$ y el $15(M=1,74)$, ambos pertenecientes al Factor 4: Otrerización, seguidos del $3(M=2)$, correspondiente al Factor 3: Negación de la identidad. 
Tabla 3. Promedio para cada pregunta del cuestionario y correspondencia con los factores propuestos por Conover

\begin{tabular}{|c|c|c|c|}
\hline Ítem & Factor Conover & $\mathbf{M}$ & SD \\
\hline $\begin{array}{l}\text { 1. ¿Con qué frecuencia la gente quita importancia a tu discapacidad o te dice que } \\
\text { podría ser peor? }\end{array}$ & F.2 Minimización & 2,9 & 1,8 \\
\hline $\begin{array}{l}\text { 2. ¿Crees que las personas sienten que necesitan ayudarte porque tienes una } \\
\text { discapacidad? }\end{array}$ & F.1 Desamparo & 2,4 & 1,7 \\
\hline $\begin{array}{l}\text { 3. ¿Crees que la gente piensa que eres menos inteligente porque tienes una } \\
\text { discapacidad? }\end{array}$ & $\begin{array}{l}\text { F.3 Negación } \\
\text { personalidad }\end{array}$ & 2,0 & 1,9 \\
\hline $\begin{array}{l}\text { 4. ¿Crees que las personas se sienten mejor cuando te ayudan porque tienes una } \\
\text { discapacidad? }\end{array}$ & F.1 Desamparo & 2,7 & 1,7 \\
\hline $\begin{array}{l}\text { 5. ¿Crees que la gente piensa que eres especial o que puedes hacer cosas } \\
\text { extraordinarias por tu discapacidad? }\end{array}$ & F.4 Otrerización & 2,2 & 1,9 \\
\hline $\begin{array}{l}\text { 6. Aunque no lo necesites o no lo hayas pedido, ¿cuántas veces la gente te ayuda o } \\
\text { pregunta si quieres ayuda por tu discapacidad? }\end{array}$ & F.1 Desamparo & 2,3 & 1,8 \\
\hline $\begin{array}{l}\text { 7. ¿Cuántas veces sientes que lo único que ven de ti es que tienes una } \\
\text { discapacidad? }\end{array}$ & $\begin{array}{l}\text { F.3 Negación } \\
\text { personalidad }\end{array}$ & 2,3 & 1,8 \\
\hline $\begin{array}{l}\text { 8. ¿Crees que las personas piensan que no puedes hacer las mismas cosas que ellas } \\
\text { porque tienes discapacidad? como tener pareja, estudiar, hacer deporte, trabajar, } \\
\text { salir o viajar. }\end{array}$ & $\begin{array}{l}\text { F.3 Negación } \\
\text { personalidad }\end{array}$ & 2,6 & 1,9 \\
\hline $\begin{array}{l}\text { 9. ¿Crees que porque tienes una discapacidad la gente te habla como si fueras un } \\
\text { niño o no te toman en serio? }\end{array}$ & $\begin{array}{l}\text { F.3 Negación } \\
\text { personalidad }\end{array}$ & 2,1 & 1,9 \\
\hline $\begin{array}{l}\text { 10. ¿Crees que la gente te ve como una persona muy madura para tu edad solo } \\
\text { porque tienes una discapacidad? }\end{array}$ & F.4 Otrerización & 1,7 & 1,7 \\
\hline $\begin{array}{l}\text { 11. Como tienes una discapacidad, ¿cuántas veces la gente quiere tomar decisiones } \\
\text { por ti sobre cosas que tú puedes decidir? }\end{array}$ & $\begin{array}{l}\text { F.3 Negación } \\
\text { personalidad }\end{array}$ & 2,2 & 1,8 \\
\hline 12. ¿Crees que la gente siente pena porque tienes una discapacidad? & F.1 Desamparo & 2,4 & 1,8 \\
\hline $\begin{array}{l}\text { 13. Como tienes una discapacidad ¿crees que la gente piensa que no puedes } \\
\text { trabajar o participar en actividades de voluntariado? }\end{array}$ & F.1 Desamparo & 2,2 & 1,9 \\
\hline $\begin{array}{l}\text { 14. Como tienes una discapacidad, ¿la gente se sorprende cuando te ve por la calle, } \\
\text { trabajando, en el cine o en un bar? }\end{array}$ & F.4 Otrerización & 2,4 & 1,9 \\
\hline $\begin{array}{l}\text { 15. Como tienes una discapacidad ¿cuántas veces crees que la gente piensa que no } \\
\text { debes salir con alguien o tener relaciones sexuales? }\end{array}$ & F.4 Otrerización & 1,7 & 1,9 \\
\hline 16. ¿La gente dice que no tendría una pareja con discapacidad? & F.4 Otrerización & 2,2 & 1,9 \\
\hline $\begin{array}{l}\text { 17. Como tienes una discapacidad ¿crees que la gente piensa que no puedes o no } \\
\text { debes tener hijos? }\end{array}$ & F.4 Otrerización & 2,0 & 2 \\
\hline 18. ¿Cuántas veces la gente te mira porque tienes una discapacidad? & F.4 Otrerización & 2,5 & 2 \\
\hline 19. ¿Cuántas veces te han dicho que tú no necesitas apoyos o adaptaciones? & F.2 Minimización & 2,2 & 1,9 \\
\hline $\begin{array}{l}\text { 20. ¿Crees que hay personas que no aceptan tu discapacidad porque no se nota y } \\
\text { dicen que eres "normal"? }\end{array}$ & F.2 Minimización & 2,6 & 2,1 \\
\hline
\end{tabular}

Fuente: elaboración propia. 
Dado que el objetivo de este trabajo es explorar las posibles diferencias en la frecuencia de las microagresiones en función de la visibilidad de la discapacidad, se llevaron a cabo análisis de diferencias tanto para los factores generales como para cada ítem. Atendiendo a las características de las distribuciones de las variables a analizar, se realizan pruebas no paramétricas (Kruskal-Wallis y, en caso de diferencia significativa, comparaciones por pares mediante Dwass-Steel-Critchlow-Fligner), utilizando, en el caso del análisis por ítems, un nivel de significación de .0025 (corrección de Bonferroni) para la determinación de diferencias globales entre los grupos de visibilidad.

Atendiendo a los resultados para los factores propuestos por Conover (2015) según el grado de visibilidad de la discapacidad, nuestros datos muestran una alta relación entre este elemento y las microagresiones recibidas. Así, la muestra con discapacidades evidentes presenta puntuaciones más altas para todos ellos, excepto para el Factor 2: "Minimización", más frecuente en la población cuya discapacidad es manifiesta en ocasiones $(M=9,2)$ o para quienes tienen una discapacidad oculta $(M=8,8)$.

Tabla 4. Promedio de la puntuación global de discriminación por factores según visibilidad de la discapacidad y comparación por pares

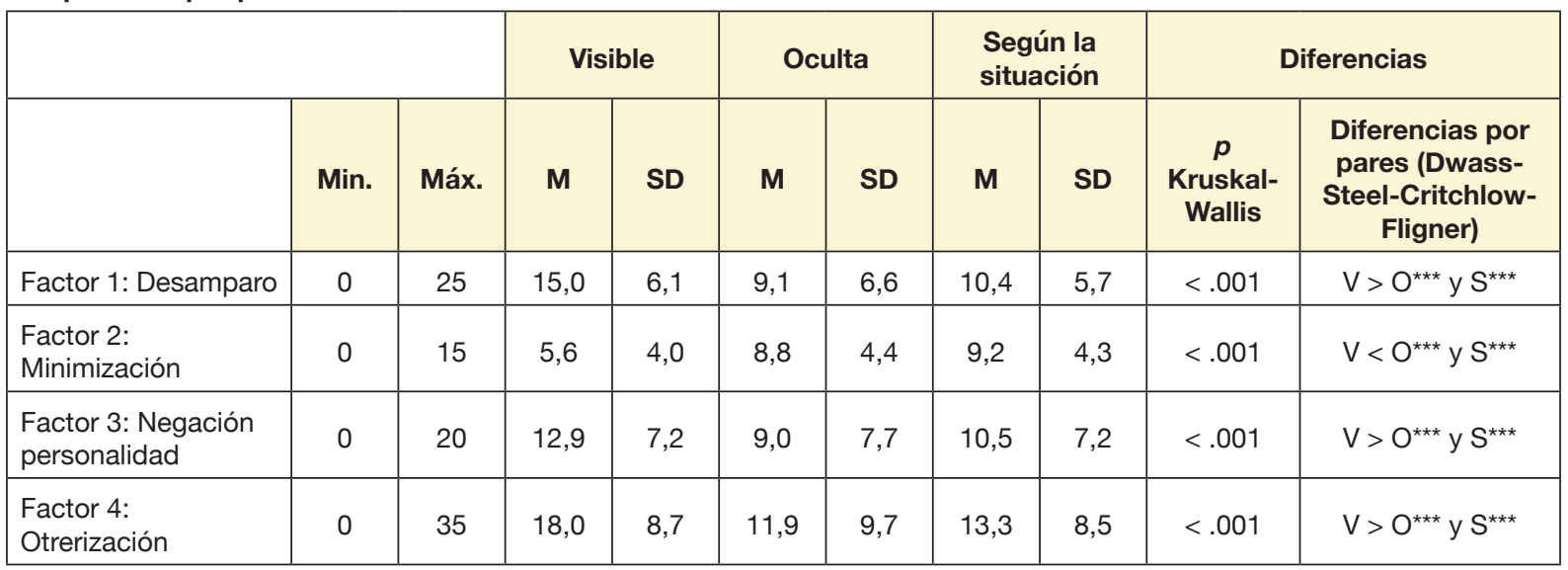

Las diferencias entre factores se codifican en forma de inicial para cada grupo, siendo "V" para Visible, "O" para Oculta y "S" para Según la situación.

${ }^{*}<.05,{ }^{* \star}<.01 \mathrm{y}^{\star \star *}<.001$

Fuente: elaboración propia.

Según los resultados para cada uno de los ítems del cuestionario, las personas con discapacidades visibles u obvias presentan puntuaciones significativamente más altas para casi todos ellos, alcanzando un promedio de 2,59, frente al grupo muestral con discapacidades ocultas $(M=1,94)$ y quienes, encontrándose en un punto intermedio en cuanto a la vinculación de la visibilidad de la discapacidad a la situación a resolver, también lo están respecto a la frecuencia en la experiencia de microagresiones capacitistas $(M=2,17)$. 


\begin{tabular}{|c|c|c|c|c|c|c|c|c|c|}
\hline & \multicolumn{2}{|c|}{ Visible } & \multicolumn{2}{|c|}{ Oculta } & \multicolumn{2}{|c|}{$\begin{array}{l}\text { Según la } \\
\text { situación }\end{array}$} & \multirow[b]{2}{*}{$\begin{array}{c}\text { M } \\
\text { Total }\end{array}$} & \multicolumn{2}{|r|}{ Diferencias } \\
\hline & $\mathbf{M}$ & SD & $\mathbf{M}$ & SD & M & SD & & $\begin{array}{l}\text { p Kruskal- } \\
\text { Wallis }\end{array}$ & $\begin{array}{l}\text { Diferencias por pares } \\
\text { (Dwass-Steel-Critchlow- } \\
\text { Fligner) }\end{array}$ \\
\hline Ítem 1 & 2,5 & 1,7 & 3,1 & 2,0 & 3,2 & 1,7 & 3,0 & $<.001$ & $V<O^{\star \star \star} y S^{\star \star \star}$ \\
\hline \multirow{2}{*}{ Ítem 2} & \multirow{2}{*}{3,2} & \multirow{2}{*}{1,7} & \multirow{2}{*}{1,6} & \multirow{2}{*}{1,6} & \multirow{2}{*}{2,0} & \multirow{2}{*}{1,6} & \multirow{2}{*}{2,3} & \multirow{2}{*}{$<.001$} & $V>O^{* * *} y S^{* * *}$ \\
\hline & & & & & & & & & $\mathrm{O}>\mathrm{S}^{\star *}$ \\
\hline Ítem 3 & 2,2 & 1,9 & 1,7 & 1,9 & 2,0 & 1,8 & 2,0 & $<.01$ & \\
\hline Ítem 4 & 3,2 & 1,6 & 2,2 & 1,9 & 2,5 & 1,7 & 2,6 & $<.001$ & $V>O^{* * *} y S^{* \star *}$ \\
\hline Ítem 5 & 2,7 & 1,8 & 1,8 & 1,9 & 2,1 & 1,9 & 2,2 & $<.001$ & $V>O^{* * *}$ y $S^{\star *}$ \\
\hline Ítem 6 & 3,1 & 1,6 & 1,6 & 1,7 & 1,8 & 1,6 & 2,2 & $<.001$ & $V>O^{\star \star *} y S^{\star \star \star *}$ \\
\hline Ítem 7 & 2,9 & 1,7 & 1,6 & 1,8 & 2,0 & 1,7 & 2,2 & $<.001$ & $V>O^{* * *} y S^{* * *}$ \\
\hline Ítem 8 & 3,2 & 1,7 & 1,9 & 1,9 & 2,3 & 1,8 & 2,5 & $<.001$ & $V>O^{\star \star \star} y S^{\star \star \star}$ \\
\hline Ítem 9 & 2,3 & 1,8 & 1,8 & 1,9 & 2,0 & 1,9 & 2,1 & $<.05$ & \\
\hline Ítem 10 & 1,8 & 1,7 & 1,5 & 1,8 & 1,6 & 1,7 & 1,7 & $<.05$ & \\
\hline Ítem 11 & 2,3 & 1,8 & 2,0 & 1,9 & 2,1 & 1,8 & 2,1 & 0.14 & \\
\hline Ítem 12 & 2,9 & 1,7 & 2,1 & 1,9 & 2,2 & 1,7 & 2,4 & $<.001$ & $V>O^{\star \star \star} y S^{\star \star \star}$ \\
\hline Ítem 13 & 2,7 & 1,8 & 1,8 & 1,9 & 1,9 & 1,8 & 2,1 & $<.001$ & $V>O^{* * \star} y^{* * *}$ \\
\hline Ítem 14 & 2,8 & 1,8 & 2,0 & 2,0 & 2,3 & 1,8 & 2,3 & $<.001$ & $V>O^{\star \star \star} y S^{\star \star \star}$ \\
\hline Ítem 15 & 2,2 & 1,9 & 1,4 & 1,9 & 1,5 & 1,8 & 1,7 & $<.001$ & $V>O^{* \star *} y S^{* \star *}$ \\
\hline Ítem 16 & 2,5 & 1,8 & 2,0 & 2,0 & 2,1 & 1,9 & 2,2 & $<.05$ & \\
\hline Ítem 17 & 2,4 & 2,0 & 1,8 & 2,0 & 1,8 & 1,9 & 2,0 & $<.001$ & $V>S^{* *}$ \\
\hline \multirow{2}{*}{ Ítem 18} & \multirow{2}{*}{3,6} & \multirow{2}{*}{1,6} & \multirow{2}{*}{1,4} & \multirow{2}{*}{1,9} & \multirow{2}{*}{2,1} & \multirow{2}{*}{1,8} & \multirow{2}{*}{2,3} & \multirow{2}{*}{$<.001$} & $V>O^{* * *} y^{* * *}$ \\
\hline & & & & & & & & & $\mathrm{O}>\mathrm{S}^{* \star *}$ \\
\hline Ítem 19 & 1,8 & 1,8 & 2,3 & 2,0 & 2,6 & 1,9 & 2,2 & $<.001$ & $\mathrm{~V}<\mathrm{S}^{\star \star *}$ \\
\hline Ítem 20 & 1,3 & 1,7 & 3,4 & 1,9 & 3,4 & 1,8 & 2,7 & $<.001$ & $V<O^{* * \star} y^{* * *}$ \\
\hline Total & 2,6 & & 1,9 & & 2,2 & & & & \\
\hline
\end{tabular}

Las diferencias entre factores se codifican en forma de inicial para cada grupo, siendo "V" para Visible, "O" para Oculta y "S" para Según la situación.

${ }^{*}<.05,{ }^{* *}<.01 \mathrm{y}^{* * *}<.001$

Fuente: elaboración propia.

Confirmando que la discriminación se fundamenta en la percepción visual de la distancia con la norma, los resultados para las preguntas relacionadas con la visibilidad de la discapacidad: el ítem 18: “¿Cuántas veces la gente te mira porque tienes una discapacidad?” y el ítem 20: “¿Crees que hay personas que no aceptan tu discapacidad porque no se nota y dicen que eres "normal"?" presentan puntuaciones que sitúan a la población en puntos claramente diferenciados según el grado de visibilidad de su discapacidad. 
Atendiendo a los datos, de manera general, el grupo con discapacidades visibles es en el que se observa mayor frecuencia de microagresiones capacitistas excepto para el ítem $20(M=1,31)$ y $19(M=1,75)$. En contraste, las puntuaciones alcanzadas por los otros dos grupos son las más elevadas para estas dos preguntas. En el caso de quienes son etiquetados como normativos-no normativos según la situación, la puntuación media alcanza un 2,62 para la pregunta 19: “¿Cuántas veces te han dicho que tú no necesitas apoyos o adaptaciones?". Para los que la discapacidad siempre es oculta, este ítem llega al 3,37. Estos ítems, junto con el ítem 1: "¿Con qué frecuencia la gente quita importancia a tu discapacidad o te dice que podría ser peor?" representan microagresiones directamente relacionadas con el cuestionamiento de las propias necesidades de apoyo y los tres conforman el Factor 2 "Minimización".

Para la población con discapacidades obvias o fácilmente identificables para terceras personas, los ítems que siguen al más reiterado están vinculados con aquellas situaciones en las que tiene lugar un ofrecimiento de ayuda, no solicitada o sostenida por una agenda oculta - consciente o no- que sirve de medio para conseguir una ganancia secundaria por prestarla. Así, los ítems 2: "¿Crees que las personas sienten que necesitan ayudarte porque tienes una discapacidad?" ( $M=3,16)$, 4: “ $i C r e e s$ que las personas se sienten mejor cuando te ayudan porque tienes una discapacidad?" $(M=3,17)$ y 6 : "Aunque no lo necesites o no lo hayas pedido, ¿cuántas veces la gente te ayuda o pregunta si quieres ayuda por tu discapacidad?" ( $M=$ $3,15)$ se encuentran en las primeras posiciones en lo que a su frecuencia se refiere; todos ellos pertenecen al Factor 1: Desamparo.

Parece, por tanto, que la visibilidad de la discapacidad es un factor determinante en cuanto a la discriminación ejercida sobre cuerpos que la expresan de manera más o menos evidente o manifiesta.

Considerando la influencia que otros factores sociodemográficos pueden ejercer en las prácticas capacitistas, los resultados obtenidos en relación con el género ${ }^{10}$ confirmarían los propuestos por la perspectiva de identidad estigmatizada primaria, desestimando que, en el caso de las microagresiones capacitistas, éste sea un factor de peso que diferencie la exposición atendiendo a la frecuencia.

10. Si bien la encuesta ofrecía tres opciones de respuesta para el apartado de género, los resultados obtenidos para la muestra que se identifica con la opción "Otro" no se añaden por ser una muestra muy escasa $(n=7)$, afectada de alto error. 


\begin{tabular}{|c|c|c|c|c|c|c|c|c|c|c|c|c|}
\hline \multirow[b]{3}{*}{ Ítem } & \multicolumn{6}{|c|}{$\begin{array}{c}\text { Mujer } \\
(n=416)\end{array}$} & \multicolumn{6}{|c|}{$\begin{array}{l}\text { Hombre } \\
(n=258)\end{array}$} \\
\hline & \multicolumn{2}{|c|}{ Visible } & \multicolumn{2}{|c|}{ Oculta } & \multicolumn{2}{|c|}{$\begin{array}{l}\text { Según la } \\
\text { situación }\end{array}$} & \multicolumn{2}{|c|}{ Visible } & \multicolumn{2}{|c|}{ Oculta } & \multicolumn{2}{|c|}{$\begin{array}{l}\text { Según la } \\
\text { situación }\end{array}$} \\
\hline & $\mathrm{M}$ & SD & $\mathrm{M}$ & SD & $\mathrm{M}$ & SD & $\mathrm{M}$ & SD & $\mathrm{M}$ & SD & $\mathrm{M}$ & SD \\
\hline 1 & 2,8 & 1,8 & 3,3 & 2,0 & 3,3 & 1,7 & 2,2 & 1,6 & 2,8 & 1,9 & 3,0 & 1,8 \\
\hline 2 & 3,2 & 1,7 & 1,5 & 1,6 & 2,1 & 1,6 & 3,1 & 1,7 & 1,6 & 1,5 & 1,9 & 1,6 \\
\hline 3 & 2,2 & 1,9 & 1,4 & 1,8 & 2,0 & 1,8 & 2,2 & 1,8 & 2,2 & 2,0 & 2,0 & 1,9 \\
\hline 4 & 3,2 & 1,6 & 2,1 & 1,8 & 2,5 & 1,7 & 3,1 & 1,7 & 2,3 & 1,9 & 2,3 & 1,6 \\
\hline 5 & 2,8 & 1,8 & 1,7 & 1,9 & 2,0 & 1,9 & 2,4 & 1,7 & 2,0 & 1,9 & 2,0 & 2,0 \\
\hline 6 & 3,3 & 1,7 & 1,6 & 1,7 & 1,8 & 1,6 & 3,0 & 1,6 & 1,6 & 1,7 & 1,7 & 1,6 \\
\hline 7 & 3,1 & 1,6 & 1,6 & 1,8 & 2,1 & 1,7 & 2,7 & 1,9 & 1,6 & 1,8 & 1,8 & 1,7 \\
\hline 8 & 3,2 & 1,7 & 1,9 & 1,9 & 2,3 & 1,8 & 3,1 & 1,7 & 1,9 & 1,9 & 2,3 & 1,9 \\
\hline 9 & 2,3 & 1,9 & 1,7 & 2,0 & 2,0 & 1,9 & 2,3 & 1,8 & 2,2 & 1,9 & 1,9 & 1,9 \\
\hline 10 & 1,9 & 1,7 & 1,5 & 1,8 & 1,7 & 1,7 & 1,7 & 1,6 & 1,8 & 1,8 & 1,3 & 1,6 \\
\hline 11 & 2,3 & 1,9 & 1,9 & 1,9 & 2,1 & 1,8 & 2,3 & 1,7 & 2,3 & 1,9 & 2,0 & 1,8 \\
\hline 12 & 3,1 & 1,7 & 2,1 & 1,8 & 2,3 & 1,8 & 2,7 & 1,6 & 1,8 & 1,9 & 1,8 & 1,6 \\
\hline 13 & 2,7 & 1,7 & 1,7 & 1,9 & 1,8 & 1,7 & 2,6 & 1,8 & 1,8 & 1,9 & 2,1 & 1,9 \\
\hline 14 & 2,9 & 1,8 & 2,0 & 2,0 & 2,4 & 1,9 & 2,6 & 1,7 & 1,9 & 2,0 & 2,0 & 1,8 \\
\hline 15 & 2,1 & 2,0 & 1,2 & 1,8 & 1,4 & 1,7 & 2,4 & 1,8 & 1,7 & 2,0 & 1,6 & 2,0 \\
\hline 16 & 2,4 & 1,8 & 1,9 & 1,9 & 2,3 & 1,8 & 2,5 & 1,7 & 2,1 & 2,2 & 1,8 & 1,9 \\
\hline 17 & 2,5 & 2,0 & 1,7 & 2,0 & 1,9 & 1,8 & 2,3 & 1,9 & 1,9 & 2,1 & 1,5 & 1,9 \\
\hline 18 & 3,7 & 1,7 & 1,3 & 1,8 & 2,1 & 1,8 & 3,4 & 1,5 & 1,6 & 1,9 & 1,9 & 1,8 \\
\hline 19 & 2,0 & 1,9 & 2,2 & 2,1 & 2,7 & 1,9 & 1,5 & 1,7 & 2,4 & 2,0 & 2,3 & 2,0 \\
\hline 20 & 1,6 & 1,9 & 3,6 & 1,8 & 3,6 & 1,7 & 0,9 & 1,4 & 2,9 & 2,0 & 3,0 & 1,9 \\
\hline Total & 2,7 & & 1,9 & & 2,2 & & 2,5 & & 2,0 & & 2,0 & \\
\hline
\end{tabular}

Fuente: elaboración propia.

\section{Discusión y conclusiones}

La discapacidad puede ser explicada como un hecho social complejo, con multitud de variaciones distintas que pueden ser resumidas o explicadas a partir de tipologías tradicionalmente estudiadas, cuyo origen se basa en categorías médicas (intelectual, física, visual, auditiva, etc.). Con menor intensidad, también se han evidenciado tradicionalmente elementos tipológicos relacionados con el momento de "emergencia" de la discapacidad, distinguiendo fundamentalmente entre congénita o sobrevenida. 
Sabemos que la experiencia de la discapacidad es muy distinta según las tipologías descritas, cuando no abiertamente contrapuestas (como es el caso de las discapacidades de la visión y la audición). Igualmente ocurre en el caso de la discapacidad congénita (asociada a una experiencia vital total) frente a sobrevenida (que implica una transformación vital súbita). En este trabajo, nos hemos planteado estudiar una tipología menos atendida, aunque no del todo inexplorada (Gonzales et al., 2015; Kattari, 2019, 2020; Kattari et al., 2018; Nario-Redmond et al., 2019; Olkin et al., 2019), como es la visibilidad de la discapacidad, es decir, la medida en que esta resulta o no evidente ante los demás.

El peso que la visibilidad de la discapacidad tiene en la experiencia de microagresiones capacitistas que presentamos en este artículo confirma que estamos ante una variable relevante para el estudio de la discriminación por discapacidad. En trabajos como el de Conover (2015), tomado como referencia para este artículo, o Nario-Redmond et al. (2019), la muestra con una discapacidad visible presenta los niveles más altos de microagresiones capacitistas, significativamente por encima de los grupos que definen su discapacidad como semi visible o para quienes la definen como invisible. También con expresiones específicas, como las identificadas en el estudio desarrollado por Wang et al. (2019) en relación con la ayuda no solicitada como una de las manifestaciones capacitistas más frecuentes dirigidas a personas con discapacidades visibles.

En relación con la invisibilidad de la discapacidad, las microagresiones se presentan en forma de cuestionamiento de la necesidad de apoyos específicos, que sí se interpretan como inevitables en el caso de personas con discapacidades visibles. Esta demanda reiterada de explicaciones en torno a los ajustes requeridos es una experiencia habitual entre quienes transitan un espacio normativo desde cuerpos que, a ojos ajenos, aparentan normalidad. Es desde este planteamiento binario a través del que se identifica al otro como normal o anormal, como sano o enfermo, válido o inválido; en él se sustentan muchas de las prácticas producidas por ese dispositivo de la discapacidad que sitúa a un grupo de población en una posición de inferioridad con respecto al hegemónico, que determina el punto de corte.

En el caso de la discapacidad, la referencia para la clasificación binaria sería la normatividad corporal. El sesgo a través del que situamos a las personas dentro de ese continuo obviaría toda la complejidad que la discapacidad misma significa, en múltiples campos y dimensiones. De esta manera, esta interpretación categórica, dicotómica, segmenta a la población entre normativa y no normativa, y para ello necesita de la inmediatez de la evidencia de la discapacidad. Así, quienes presentan discapacidades ocultas o dependientes de la demanda del contexto para su identificación, pueden sortear un primer filtro de ese juicio sumario que les ubica próximos a la normatividad, incluso ser más cercanos al centro de ese eje del continuo normativo que en el caso de todos aquellos cuerpos con discapacidades fácilmente identificables.

En los dos extremos del eje visiblidad-invisibildad que segmenta el campo social que produce y reproduce microagresiones capacitistas, se encuentran quienes difícilmente pueden escapar a la identificación como seres no normativos y que soportan altos niveles de discriminación, y quienes, por pasar inadvertidos en toda situación o dependiendo de ésta, reciben otro tipo de microagresiones en grado y forma, que requieren de especial atención. Si en la producción cognitiva de juicios sumarios hacia otras personas también media el sesgo binario, a través del que se evalúa la nueva información desde un planteamiento del "todo o nada", las discapacidades invisibles $-\mathrm{y}$, con más razón, aquellas dependientes de la demanda del entorno-, serán, probablemente, interpretadas como datos contradictorios, por lo que la comprensión de lo que supone o significa una discapacidad no evidente podría acercarse a la experiencia de una disonancia cognitiva que lleve a la negación o minimización de la discapacidad misma. 
Pero de la misma manera se sabe que, incluso en actitudes disposicionales primarias basadas en codificaciones binarias de la realidad, la influencia del contexto es fundamental para el ajuste equilibrado del pensamiento dicotómico (Ninivaggi, 2015). Por tanto, el trabajo de sensibilización y reformulación de actitudes negativas hacia personas con discapacidad debe abordarse desde el entorno escolar y familiar, con el objetivo de suavizar la tendencia a posiciones polarizadas durante los primeros años de desarrollo, que puedan perdurar a lo largo de la vida. También desde los medios de comunicación, pues participan de manera significativa en la representación de la discapacidad como reproductores de los marcos centrales que explican lo que la discapacidad significa (modelo médico, social o de la diversidad), los dispositivos de encuadre que dichos marcos ponen en funcionamiento y los dispositivos de razonamiento que promueven (Goethals et al., 2020).

La convivencia con la diferencia es una oportunidad para conocer otras formas de relacionarse, interpretar e interactuar con el mundo, pero la inclusión requiere de intervención consciente. El abordaje de la discapacidad -como de otras diferencias tendentes a ser leídas desde el prejuicio-, necesita de perspectivas que generen espacios abiertos para el pensamiento crítico. Trabajos como el de Mara Sapon-Shevin (2017) muestran la importancia de comprender la complejidad de la diferencia, así como de reconocer la discriminación que algunos grupos sociales sufren, como requisitos para convertirnos en aliados activos contra las desigualdades. Proporcionar palabras para nombrar e identificar los prejuicios y proveer de respuestas específicas cuando se manifiestan, permite romper con el sesgo binario y, por tanto, acercarnos a una acogida más honesta de la diversidad.

Los resultados obtenidos en este estudio aclaran ciertas distancias y puntos de encuentro en lo que a la expresión de las microagresiones capacitistas en nuestro contexto se refiere, atendiendo a la evidencia o el ajuste de la población con discapacidad a la norma. Este hecho adelanta la necesidad de estudios específicos para profundizar en ellas, así como en la identificación de los distintos elementos y agentes involucrados en lo que Foucault (1991) denomina dispositivo y Contino (2013) recoge y propone como marco para abordar la discapacidad y localizar esas fisuras o líneas de fuga, necesarias para indagar acerca de planteamientos novedosos sobre lo que habitar cuerpos no normativos supone y quién y cómo se define.

Dos cuestiones que considerar de cara a la mejora del instrumento empleado para este estudio tienen que ver, por un lado, con el diseño de este y por otro con garantizar la accesibilidad a la población con dificultades de comprensión. En el primer caso, si bien nuestro objetivo era adaptar la escala original siendo lo más fieles posible a su sintaxis y el significado de los ítems originales, es cierto que en líneas futuras de desarrollo se podría reforzar el formulado de las preguntas centrándolo en la observación objetiva de microagresiones y no tanto en la percepción sobre lo que piensan los otros (aunque no lo manifiesten). Por otro lado, tal y como nos lo trasladaron algunas personas que facilitaron la cumplimentación de la encuesta por participantes con discapacidad intelectual, la elaboración de una versión de la misma en lectura fácil resulta imprescindible para asegurar la representación de una muestra extensa de este grupo poblacional.

Es necesario ampliar la investigación cuantitativa en lo que al abordaje de las microagresiones capacitistas se refiere, sin olvidar el enfoque cualitativo para considerar las consecuencias que supone estar expuesto, de manera continuada, a una discriminación extendida a prácticamente todas las esferas de actividad.

Determinadas variables, como el momento de aparición de la discapacidad, el empleo de ayudas técnicas o humanas, el nivel de estudios alcanzado o el desarrollo de la conciencia de pertenencia a un grupo 
minoritario y discriminado, pueden tener una relación significativa con la variedad y frecuencia con la que se es víctima de microagresiones capacitistas. Es necesario diseñar futuras investigaciones considerando esta relación en el constructo "capacitismo", atendiendo a las diferencias entre aquellos cuerpos que, encontrándose dentro de la normatividad la ven interrumpida; participan en grupos autogestionados a través de los que concienciarse de la opresión compartida, o las implicaciones que el uso de asistencia para la resolución de las necesidades de apoyo tiene en la visibilidad de la discapacidad. También en cuanto a la profundización de la comprensión del capacitismo interiorizado (Campbell, 2008; Dunn, 2019) entre quienes nacen y son ubicados en los márgenes de la normatividad y quienes se ven expulsados tras una discapacidad adquirida. La actual situación de crisis sanitaria provocada por el COVID-19 y todas las medidas relacionadas con las decisiones en lo que a la salud se refiere, pero también a aquellas relacionadas con la consideración de las especificidades en la atención a las necesidades de apoyo de las personas con discapacidad que ha puesto de manifiesto, puede resultar una oportunidad única para el estudio del dispositivo de la discapacidad.

En línea con las conclusiones que aportan los estudios que contemplan la visibilidad de la discapacidad como factor determinante e involucrado en la discriminación capacitista, parece conveniente que su estudio se diseñe en relación con las posibilidades de ajuste a un funcionamiento normativo como medida de todas las cosas. La aproximación al fenómeno descarta planteamientos más tradicionales, como aquellos basados en el tipo de discapacidad, por ejemplo, como factores determinantes para el tema que nos ocupa. El objeto de la discriminación capacitista parece no ser solo el "cuerpo discapacitado", también el "cuerpo discapacitado disimulado", incluso con los apoyos necesarios para el ajuste eficaz. 


\section{Referencias bibliográficas}

Ashraf, S. et al. (2018). "Violence against Women with Disabilities: A Qualitative Investigation”. Annals of King Edward Medical University, 23, pp. 540-545. DOI: https://doi.org/10.21649/akemu.v23i4.2234.

Baltar, F. y Gorjup, M. T. (2012). "Muestreo mixto online: una aplicación en poblaciones ocultas”. Intangible Capital, 8(1), pp. 123-149. DOI: http://dx.doi.org/10.3926/ic.294.

Bell, A. K. (2015). Nothing about us without us: A qualitative investigation of the experiences of being a target of ableist microaggressions. ProQuest Information \& Learning.

Bogart, K. R. y Dunn, D. S. (2019). “Ableism Special Issue Introduction”. Journal of Social Issues, 75(3), pp. 650664. DOI: https://doi.org/10.1111/josi.12354.

Campbell, F. A. K. (2001). "Inciting legal fictions: Disability's date with ontology and the ableist body of the law". Griffith Law Review, 10, pp. 42-62.

Campbell, F. A. K. (2008). "Exploring internalized ableism using critical race theory". Disability \& Society, 23(2), pp. 151-162. DOI: https://doi.org/10.1080/09687590701841190.

Campbell, F. A. K. (2009). Contours of ableism: the production of disability and abledness. Australia: Palgrave MacMillan.

Candela, S. y Mulet, G. (2018). "Mujeres, diversidad funcional y multidiscriminación”. Journal of Feminist, Gender and Women Studies, 7, pp. 45-56. DOI: http://dx.doi.org/10.15366/jfgws2018.7.005.

Chambers, C. R. (2011). "Candid Reflections on the Departure of Black Women Faculty from Academe in the United States". Negro Educational Review, 62/63(1-4), pp. 233-260,265.

Conover, K. J. (2015). Ableist microaggressions scale: Development, validation, and relationship with social support (Ph.D.). ProQuest Dissertations and Theses. Ann Arbor: University of California, Santa Barbara.

Conover, K. J. et al. (2017). "Development and validation of the Ableist Microaggressions Scale". The Counseling Psychologist, 45(4), pp. 570-599. DOI: https://doi.org/10.1177/0011000017715317.

Contino, A. M. (2013). "El dispositivo de discapacidad”. Tesis Psicológica: Revista de la Facultad de Psicología, $8(1)$, pp. 174-183.

Deal, M. (2007). "Aversive disablism: subtle prejudice toward disabled people”. Disability \& Society, 22(1), pp. 93107. DOI: https://doi.org/10.1080/09687590601056667.

Dirth, T. P. y Branscombe, N. R. (2018). "The social identity approach to disability: Bridging disability studies and psychological science”. Psychological Bulletin, 144(12), pp. 1300.

Dirth, T. P. y Branscombe, N. R. (2019). "Recognizing ableism: A social identity analysis of disabled people perceiving discrimination as illegitimate”. Journal of Social Issues, 75(3), pp. 786-813. DOI: https://doi.org/10.1111/ josi. 12345.

Dunn, D. S. (2019). "Outsider Privileges Can Lead to Insider Disadvantages: Some Psychosocial Aspects of Ableism". The Journal of Social Issues, 75(3), pp. 665-682. DOI: https://doi.org/10.1111/josi.12331.

Dunn, D. S. y Andrews, E. E. (2015). 'Person-first and identity-first language: Developing psychologists' cultural competence using disability language". American Psychologist, 70(3), pp. 255-264. DOI: https://doi. org/10.1037/a0038636. 
España. Instrucción de 19 de marzo de 2020 del Ministerio de Sanidad, por la que se establecen criterios interpretativos para la gestión de la situación de crisis sanitaria ocasionada por el COVID-19, Boletín Oficial del Estado, 20 de marzo de 2020, núm. 76, pp. 26311-26312.

Fisher, M. y Keil, F. C. (2018). "The Binary Bias: A Systematic Distortion in the Integration of Information”. Psychological Science, 29(11), pp. 1846-1858. DOI: https://doi.org/10.1177/0956797618792256.

Forber-Pratt, A. J. et al. (2017). "Disability identity development: A systematic review of the literature". Rehabilitation Psychology, 62(2), pp. 198-207. DOI: https://doi.org/10.1037/rep0000134.

Foucault, M. (1991). “El juego de Michel Foucault”. En F. Alvarez-Uria y J. Varela (eds.), Saber y verdad (pp. 62-93) Madrid: Ediciones de la Piqueta.

Friedman, C. (2016). Aversive ableism: Subtle discrimination and prejudice towards disabled people. Chicago: University of Illinois.

Friedman, C. (2017). "Siblings of People with Disabilities' Explicit and Implicit Disability Attitude Divergence". Journal Of Social Work In Disability \& Rehabilitation, 16(1), pp. 74-92. DOI: https://doi.org/10.1080/153671 OX.2017.1260519.

Gill, C. J. (1997). "Four types of integration in disability identity development". Journal of Vocational Rehabilitation, 9, pp. 39-46. DOI: https://doi.org/10.1016/S1052-2263(97)00020-2.

Goethals, T. et al. (2020). "I am not your metaphor: frames and counter-frames in the representation of disability". Disability \& Society, 1-19. DOI: https://doi.org/10.1080/09687599.2020.1836478.

Goffman, E. (1970). Estigma : la identidad deteriorada. 1a. ed., 3a. reimp. Buenos Aires: Amorrortu.

Gonzales, L. et al. (2015). "Microaggressions experienced by persons with mental illnesses: An exploratory study". Psychiatric Rehabilitation Journal, 38(3), pp. 234-241. DOI: https://doi.org/10.1037/prj0000096.

Grier-Reed, T. L. (2010). "The African American Student Network: Creating Sanctuaries and Counterspaces for Coping With Racial Microaggressions in Higher Education Settings”. Journal of Humanistic Counseling, Education \& Development, 49(2), pp. 181-188.

Harder, J. A. et al. (2019). "Demographic, Experiential, and Temporal Variation in Ableism”. Journal of Social Issues, 75(3), pp. 683-706. DOI: https://doi.org/10.1111/josi.12341.

Harris, A. y Wideman, D. (1988). "The Construction of Gender and Disability in Early Attachment”. En M. Fine y A. Asch (eds.), Women with disabilities: essays in psychology, culture, and politics, Health, society, and policy (pp. 115-138). Philadelphia: Temple University Press.

Hernández, M. A. (2018). "Contractualismo y discapacidad. Hacia una crítica del capacitismo y el contrato funcional desde la obra de Carole Pateman”. Hybris: revista de filosofía, 9(1), pp. 295-322.

Hill Collins, P. y Bilge, S. (2019). Interseccionalidad. Madrid: Ediciones Morata.

Hooks, B. (1990). Yearning: Race, gender, and cultural politics. Boston: MA: South End Press.

Hutcheon, E. J. y Wolbring, G. (2012). "Voices of «disabled» post secondary students: Examining higher education «disability» policy using an ableism lens". Journal of Diversity in Higher Education, 5(1), pp. 39-49. DOI: https:// doi.org/10.1037/a0027002.

Jiménez, A. y Huete, A. (2010). "Políticas públicas sobre discapacidad en España. Hacia una perspectiva basada en los derechos". Política y Sociedad, 47(1), pp. 137-152. 
Kattari, S. K. (2019). "The Development and Validation of the Ableist Microaggression Scale". Journal of Social Service Research, 45(3), pp. 400-417. DOI: https://doi.org/10.1080/01488376.2018.1480565.

Kattari, S. K. (2020). "Ableist Microaggressions and the Mental Health of Disabled Adults". Community Mental Health Journal, 56(6), pp. 1170-1179. DOI: https://doi.org/10.1007/s10597-020-00615-6.

Kattari, S. K. et al. (2018). "«you look fine!»: Ableist experiences by people with invisible disabilities”. Affilia: Journal of Women \& Social Work, 33(4), pp. 477-492. DOI: https://doi.org/10.1177/0886109918778073.

Keller, R. M. y Galgay, C. E. (2010). "Microaggressive experiences of people with disabilities”. En D. W. Sue (ed.), Microaggressions and marginality: Manifestation, dynamics, and impact. (pp 241-267). Hoboken, NJ: John Wiley \& Sons Inc.

Lett, K. et al. (2018). "Impact of ableist microaggressions on university students with self-identified disabilities". Disability \& Society 35(1): pp. 1-16.

Levin, S. et al. (2002). "Perceived discrimination in the context of multiple group memberships". Psychological Science, 13(6), pp. 557-560. DOI: https://doi.org/10.1111/1467-9280.00498.

Marín, C. (2013). El derecho a la propia discapacidad: el régimen de la discapacidad de obrar. Madrid: Editorial Universitaria Ramón Areces.

Martín, U. y González-Rábago, Y. (2019). "Metodología cualitativa para enfocar la mirada cuantitativa: la experiencia de los pretest cognitivos aplicados a la Encuesta de Salud del País Vasco". Empiria. Revista de metodología de ciencias sociales, 43. DOI: https://doi.org/10.5944/empiria.43.2019.24302.

Moscoso, M. y Arnau, S. (2016). "Lo Queer y lo Crip, como formas de re-apropiación de la dignidad disidente. Una conversación con Robert McRuer”. ILEMATA, Año 8(20), pp. 137-144.

Mwangi, C. A. G. et al. (2018). "Collegians Creating (Counter)Space Online: A Critical Discourse Analysis of the I, Too, Am Social Media Movement”. Journal of Diversity in Higher Education, 11(2), pp. 146-163. DOI: https:// doi.org/10.1037/dhe0000054.

Nario-Redmond, M. R. (2010). "Cultural stereotypes of disabled and non-disabled men and women: Consensus for global category representations and diagnostic domains". British Journal of Social Psychology, 49(3), pp. 471-488. DOI: https://doi.org/10.1348/014466609X468411.

Nario-Redmond, M. R. et al. (2019). "Hostile, Benevolent, and Ambivalent Ableism: Contemporary Manifestations". The Journal of Social Issues, 75(3), pp. 726-756. DOI: https://doi.org/10.1111/josi.12337.

Ninivaggi, F. J. (21 junio, 2015). Two-ness:" the Mind's Binary Code [Artículo en Psychology Today]. Recuperado de https://bit.ly/37QNE6v.

Oliver, M. (1990). The politics of disablement. Critical texts in social work and the welfare state. Basingstoke: Palgrave Macmillan.

Olkin, R. et al. (2019). "The experiences of microaggressions against women with visible and invisible disabilities". Journal of Social Issues, 75(3), pp. 757-785. DOI: https://doi.org/10.1111/josi.12342.

Otaola, M. P. y Huete, A. (2019)." Capacitismo: un fenómeno sociodemográfico”. Actas de coordinación sociosanitaria, 25, pp. 179-198.

Palombi, B. J. (2012). "Women with Disabilities: The Cultural Context of Disability, Feminism, Able-Bodied Privilege, and Microaggressions". En E. N. Williams y C. Z. Enns (eds.), The Oxford Handbook of Feminist Multicultural 
Counseling Psychology (pp 199-220). New York, NY: Oxford University Press. DOI: https://doi.org/10.1093/ oxfordhb/9780199744220.013.0011.

Pierce, C. et al. (1978). "An Experiment in Racism: TV commercials”. En C. Pierce (ed.), Television and education (pp 62-88). Beverly Hills: CA: Sage. Recuperado de http://keever.us/pierce.html.

Platero, R. y Guzmán, P. (2014). "The critical intersections of disability and non-normative sexualities in Spain". Annual Review of Critical Psychology, 11, pp. 357-387.

Purdie-Vaughns, V. y Eibach, R. P. (2008). "Intersectional invisibility: The distinctive advantages and disadvantages of multiple subordinate-group identities". Sex Roles: A Journal of Research, 59(5-6), pp. 377-391. DOI: https:// doi.org/10.1007/s11199-008-9424-4.

Sanmiquel-Molinero, L. y Pujol-Tarrés, J. (2020). "Putting emotions to work: the role of affective disablism and ableism in the constitution of the dis/abled subject". Disability \& Society, 35(4), pp. 542-569. DOI: https://doi. org/10.1080/09687599.2019.1650719.

Sapon-Shevin, M. (2017). "On the Impossibility of Learning "Not to See": Colorblindness, Invisibility, and Anti-Bias Education”. International Critical Childhood Policy Studies Journal, 6(1), pp. 38-51.

Schaff, M. (2017). Experiences of microaggressions among women with apparent disabilities: A look at the intersection of gender and disability. ProQuest Information \& Learning.

Singleton, K. (2013). "Review of Microaggressions in everyday life: Race, gender, and sexual orientation”. Psychoanalytic Psychology, 30(4), pp. 680-685. DOI: https://doi.org/10.1037/a0033932.

Solorzano, D. et al. (2000). "Critical Race Theory, racial microaggressions, and campus racial climate: the experiences of african american college students". The Journal of Negro Education, 69(1/2), pp. 60-73.

Stein, A. y Plummer, K. (1994). “«I Can’t Even Think Straight» «Queer» Theory and the Missing Sexual Revolution in Sociology". Sociological Theory, 12(2), pp. 178-187. DOI: https://doi.org/10.2307/201863.

Sue, D. W. (2010). Microaggressions in everyday life: Race, gender, and sexual orientation. Hoboken, NJ: John Wiley \& Sons Inc.

Toboso, M. (2017). "Capacitismo (Ableism)”. En L. Platero et al. (eds.), Barbarismos queer y otras esdrújulas (pp 73-81). Barcelona: Bellaterra.

Toboso, M. (2018). "Diversidad funcional: hacia un nuevo paradigma en los estudios y en las políticas sobre discapacidad/Functional diversity: towards a new paradigm in disability studies and disability policies (MISCELANEA)". Politica y Sociedad, 55(3), pp. 783. DOI: https://doi.org/10.5209/POSO.56717.

Toboso, M. y Guzman, F. (2010). "Cuerpos, capacidades, exigencias funcionales... y otros lechos de Procusto1/ Bodies, Abilities, Functional Requirements... and Others Procrustean Beds". Política y Sociedad, 47(1), pp. 67-83, 243,246.

United States (2009). Americans with Disabilities Act of 1990 (ADA). Recuperado de https://www.ada.gov/pubs/ adastatute08.htm\#12102.

Vivas, I. (2020). "Las personas con discapacidad y sus familias ante las crisis sanitarias". En E. Atienza y J. F. Rodríguez (eds.), Las respuestas del derecho a las crisis de salud pública (pp 155-174). Madrid: Dykinson.

Wang, K. et al. (2019). "Consequences of Confronting Patronizing Help for People with Disabilities: Do Target Gender and Disability Type Matter?”. Journal of Social Issues, 75(3), pp. 904-923. 
Wells, C. (2013). "Microaggressions in the Context of Academic Communities". Seattle Journal for Social Justice, 12(2), pp. 319-348.

Wolbring, G. (2008). "The Politics of Ableism". Development, 51(2), pp. 252-258. DOI: https://doi.org/10.1057/ dev.2008.17.

Yosso, T. J. et al. (2009). "Critical race theory, racial microaggressions, and campus racial climate for Latina/o undergraduates". Harvard Educational Review, 79(4), pp. 659-690. DOI: https://doi.org/10.17763/ haer.79.4.m6867014157m707I. 